\title{
Genießen islamistische Parteien tatsächlich einen politischen Vorteil? Das Beispiel Tunesien
}

\author{
Tim Lewis Poppenborg ${ }^{1}$, Bernd Schlipphak ${ }^{2, *}$ \\ 1 London School of Economics, London, Großbritannien \\ 2 Universität Münster, Institut für Politikwissenschaft, Münster, Deutschland \\ * E-Mail: bernd.schlipphak@uni-muenster.de
}

\section{Zusammenfassung}

Ein kürzlich erschienener Literaturüberblick von Cammett und Luong diskutiert den politischen Vorteil islamistischer Parteien in Nordafrika und Nahost, kann die angenommene Funktionsweise des politischen Vorteils aber nicht empirisch belegen. Durch den Rückgriff auf das Konzept der individuellen Nutzung von Heuristiken umgehen wir gängige Messprobleme und argumentieren, dass durch die von islamistischen Parteien bereitgestellten „cues“ bestimmte Gruppen von BürgerInnen eine höhere Wahlwahrscheinlichkeit zugunsten eben dieser Parteien haben sollten. Der Test unseres Arguments mit Daten des Arab Barometer für das Beispiel Tunesien zeigt ein ambivalentes Bild. Während Befragte, die unverbrauchte politische Akteure bevorzugen, zu islamistischen Parteien tendieren, gilt dies für Befragte, denen die Integrität von KandidatInnen wichtig ist, entgegen unserer Erwartungen nicht.

\section{Schlüsselwörter}

politischer Vorteil, Islamismus, Naher Osten, Heuristiken, Wahlverhalten

\section{Do Islamist parties actually enjoy a political advantage? The Tunisian case}

\begin{abstract}
A recent literature review by Cammett and Luong discusses the political advantage of Islamist parties in North Africa and the Middle East. Yet, there is a lack of empirical evidence with regard to the mechanism through which the political advantage is assumed to work. Drawing on the concept of the individual use of heuristics, we are able to circumvent common measuring problems. We then argue that specific groups of citizens should have a higher likelihood to vote for Islamist parties as a result of the cues these parties provide. Testing our argument with data from the Arab Barometer for the Tunisian case, we find mixed results. In line with our expectations, respondents who prefer untested political actors are more likely to vote for Islamist parties, whereas - contrary to our hypothesis - respondents who value integrity are less likely to vote for these parties.
\end{abstract}

\section{Keywords}

political advantage, Islamism, Middle East, heuristics, voting behavior

The authors have declared that no competing interests exist. 


\section{Einleitung}

Als der sogenannte „Arabische Frühling“ in den Jahren 20IO/2OII zu einer politischen Öffnung einiger mehrheitlich muslimischer Länder führte, profitierten hiervon vor allem islamistische Parteien. ${ }^{I}$ Damit schien sich zu bewahrheiten, was als "Islamist dilemma“ (Hamid 20II, 40) jahrelang die US-amerikanische Außenpolitik geprägt hatte. Demnach sei es für die USA unmöglich die Demokratisierung der arabischen Welt zu fördern, ohne die Machtübernahme durch IslamistInnen zu riskieren. ${ }^{2}$ Auch die Europäische Kommission räumte ein, sich von autokratischen Regimen politische Stabilität erhofft und in islamistischen Parteien eine Gefahr für eine mögliche Demokratisierung gesehen zu haben (Phillips 20II). Westliche Medien wiederum fragten sich angesichts der islamistischen Wahlsiege, ob auf den „Arabischen Frühling" nicht zwangsläufig ein "Islamist winter" folge (Washington Times 20II; Münch 20I2; Steinvorth 20I2). Islamistischen Parteien wurde also von verschiedenen Seiten ein politischer Vorteil eingeräumt, der aufgrund der häufigen Assoziierung des Islamismus mit einervon Natur aus gewaltsamen und antiwestlichen Ideologie negativ konnotiert ist (Ayoob 2004, I; Brown et al. 2006, 3). Doch genießen islamistische Parteien diesen politischen Vorteil tatsächlich?

Die politischen Ereignisse in den arabischen Ländern in der jüngeren Vergangenheit lassen - anders als die konstituierenden Wahlen von 2011/2012 in Tunesien und Ägypten - Zweifel an dieser These aufkommen. In den im Oktober 20I4 abgehaltenen Parlamentswahlen in Tunesien erhielt die säkulare Sammlungspartei Nidaa Tounes rund $39 \%$ der Sitze und lag damit rund acht Prozentpunkte vor der islamistischen Ennahda (SZ 2OI4). Allein durch die Betrachtung von Wahlergebnissen lässt sich demnach nicht ermitteln, ob für islamistische Parteien in Transformationsstaaten tatsächlich ein politischer Vorteil existiert. In unserem Aufsatz gehen wir daher zum ersten Mal anhand einer systematischen empirischen Analyse der Frage nach, ob islamistische Parteien in arabischen Ländern einen politischen Vorteil gegenüber konkurrierenden Parteien im Hinblick auf die Wahrscheinlichkeit ihrer Wahl durch die BürgerInnen haben.

I Aufgrund der Vielfältigkeit und den unterschiedlichen Entwicklungsrichtungen islamistischer Parteien verwenden wir eine weite Definition des Begriffs politischer Islam bzw. Islamismus und verstehen diesen als a form of instrumentalization of Islam by individuals, groups and organizations that pursue political objectives. It provides political responses to today's societal challenges by imagining a future, the foundations for which rest on reappropriated, reinvented concepts borrowed from the Islamic tradition" (Denoeux 2002, 6I).

2 Bei den Wahlen zur Verfassungsgebenden Versammlung (2OII) wurde Tunesiens islamistische Ennahda mit rund 37\% der Stimmen stärkste Kraft (Bustos 20II, 5). Bei den ägyptischen Unterhauswahlen (20II/2OI2) gewannen die mit der Muslimbruderschaft assoziierten Parteien und die salafistisch-islamistische Al Nour die absolute Mehrheit der Stimmen (kumuliert 63\%) (ICG 2012). In Marokko wurde bei den vorgezogenen Parlamentswahlen (2OII) die islamistische Partei für Gerechtigkeit und Entwicklung mit rund 27\% der Sitze stärkste Kraft (Economist 201I)
Cammett und Luong (2014) haben in der Literatur drei separate Argumentationsstränge identifiziert, die von AutorInnen genutzt werden, um die Massenzustimmung islamistischer Parteien zu erklären. Manche nehmen an, die Ideologie des politischen Islam sei den übrigen säkularen Ideologien überlegen. Andere AutorInnen verweisen darauf, dass sich IslamistInnen über das Erzeugen sozialer Wohlfahrt eine außergewöhnlich starke soziale Basis erarbeitet hätten. Wiederum andere meinen, der Islamismus sei besser organisiert als andere politische Bewegungen (Cammett/Luong 2014, I88, 193-99). Dass die konkreten Politiken von IslamistInnen die Quelle eines politischen Vorteils darstellen könnten, wird dabei größtenteils nicht in Betracht gezogen bzw. sogar explizit verneint (Pepinsky et al. 20I2). Das Problem der Untersuchung des vermeintlichen politischen Vorteils islamistischer Parteien ist in erster Linie ein Messproblem, das sich aus zwei Punkten ergibt. Wie können - erstens - relative Stärken der IslamistInnen in den Bereichen Ideologie, soziale Basis und organisatorische Kapazitäten erfasst werden? Selbst dort, wo aussagekräftige Indikatoren bestünden, fehlt das nötige Datenmaterial (Cammett/Luong 20I4, I93f., 202). Zweitens erklärt die bloße Existenz solcher Stärken noch nicht, wie diese den Wahlentscheid der WählerInnen in mehrheitlich muslimisch geprägten Ländern zugunsten islamistischer Parteien beeinflussen könnten.

Um diese Probleme zu überwinden folgen wir in diesem Artikel einem alternativen Zugang in der Forschung, der eine empirisch-quantitative Analyse des politischen Vorteils ermöglicht. Wir argumentieren, dass islamistische Parteien ihren Wählern einen „information shortcut" oder "cue" als Heuristik ${ }^{3}$ bereitstellen, der die von ihnen zu erwartenden moralischen Qualitäten (Cammett / Luong 20I4) bzw. die damit verbundenen politischen Positionen (Pepinsky et al. 2012) signalisiert und so bei WählerInnen positive Evaluierungen auslöst.

In der Folge führen wir zunächst kurz in die Literatur zu politischen Heuristiken ein. Dann diskutieren wir zum einen, inwiefern die religiöse Orientierung einer Partei dieser Chancen eröffnet, Heuristiken zu generieren, die säkularen Parteien nicht zur Verfügung stehen. Zum anderen erläutern wir das Argument des politischen Vorteils islamistischer Parteien, wie es Cammett und Luong (2014) im Ergebnis ihres Literaturüberblicks darstellen. Anschließend führen

3 Heuristiken können definiert werden als „problem-solving strategies (often employed automatically or unconsciously) which serve to ,keep the information processing demands of the task within bounds"“ (Lau/Redlawsk 200I, 952). Sie sind Hilfestellungen, die WählerInnen ermöglichen sollen, mit geringem kognitivem Aufwand bestmögliche Entscheidungen zu treffen. 
wir beide Forschungsstränge zusammen und leiten aus der Diskussion unsere forschungsleitenden Hypothesen ab. Dabei erwarten wir, dass das islamistische Parteilabel - d.h., die (Selbst-)Bezeichnung einer Partei als islamistisch oder mit einer islamistischen Programmatik versehen - potentielle WählerInnen über konkrete Kandidatenspezifika in Hinblick auf deren moralische und politische Qualitäten informiert.

Wir überprüfen diese Erwartungen im empirischen Teil des Artikels anhand der tunesischen Daten aus der aktuellen Welle des Arab Barometers. Unsere Resultate zeigen, dass die für unser Argument ausgewählten Indikatoren signifikante und substantiell relevante Effekte auf die individuelle Wahrscheinlichkeit haben, eine islamistische Partei zu wählen. Abschließend diskutieren wir die Implikationen und die Beschränktheit unserer Ergebnisse und weisen auf - vornehmlich methodologische - Forschungsdesiderata hin.

\section{Der politische Vorteil islamistischer Parteien als Heuristik}

Damit Menschen die Komplexität ihrer Umwelt bewältigen können, greifen sie ständig auf Heuristiken zurück. Sie stellen Strategien dar, mithilfe derer die Klassifikation von Informationen vereinfacht wird. Die Nutzung von Heuristiken erfolgt typischerweise unbewusst und geht zurück auf „well learned - perhaps even genetic cognitive predispositions" (Lau/Redlawsk 2006, 23I). Politische Akteure können sich diesen Umstand zu Nutze machen, indem sie potentiellen WählerInnen entsprechende Heuristiken anbieten und damit beeinflussen, wie Informationen klassifiziert werden (Rahn I993, 472f.; Lau/Redlawsk 200I, 952).

\subsection{Literaturüberblick}

Das Interesse der Politikwissenschaft an Heuristiken geht auf die Frage zurück, warum Bürger in Demokratien trotz ihres relativen Desinteresses an Politik und einer damit einhergehenden systematischen Unwissenheit über die Positionen politischer Akteure in der Tendenz dennoch jene KandidatInnen und Parteien wählen (können), die ihren tatsächlichen Präferenzen entsprechen. Die gemeinsame Prämisse vieler verschiedener Ansätze bildet dabei die Annahme, dass der Mensch ein „cognitive miser" (Lau/Sears 1986, 349) ist, der schon mit wenigen Informationen über den Rückgriff auf Heuristiken rationale Entscheidungen treffen kann. Dieses von Autoren wie Samuel Popkin (199I, aber siehe bereits Simon 1957) als "low information rationality“ in die politische Verhaltensforschung eingeführte Paradigma hat sich Lau und Redlawsk (200I, 952) zufolge rasch zu einem Gemeinplatz entwickelt.
Die Heuristiken, die WählerInnen zur Verfügung stehen, sind zahlreich. Neben den vornehmlich verwendeten ideologischen cues - Hinweise auf grundlegende Positionen von Parteien ${ }^{4}$ - sind insbesondere Parteistereotypen von großer Bedeutung. Parteistereotype, d.h. "those cognitive structures that contain citizens' knowledge, beliefs, and expectations about (...) political parties" (Rahn 1993, 474) sind von besonderer Relevanz für unsere Fragestellung und gelten als vielgenutzte Heuristiken, nicht zuletzt aufgrund ihrer großen kognitiven Ersparnis. Diese liegt darin begründet, dass von diesen Heuristiken ausgehend WählerInnen auf die Positionierung von Parteien und KandidatInnen in Bezug auf zahlreiche politische Einzelthemen zurückschließen können. Fehlerhafte Annahmen auf Seiten von WählerInnen entstehen immer dann, wenn Parteien und KandidatInnen eine Politik betreiben, die sich vom Stereotyp unterscheidet. Dementsprechend ist die Entstehung und Effektivität von Stereotypen von einer gewissen Konstanz abhängig, was die Präsenz, Ausrichtung und Interaktion von politischen Parteien betrifft (Lau/Redlawsk 200I, 953; 2006, 232; Rahn I993, 473f.).

\subsection{Die Rolle religiöser Heuristiken}

Erst seit wenigen Jahren setzen sich Untersuchungen zunehmend mit der Frage auseinander, welche Effekte die Religiosität von politischen KandidatInnen und Parteien auf deren Evaluierung durch potentielle WählerInnen hat - d.h., welche Rolle der Religiosität einer Partei als Heuristik zukommt.

Calfano und Djupe (2009) analysierten in ihrer experimentellen Studie den sogenannten "Code" der republikanischen Partei in den USA. Dieser Code beschreibe eine Strategie, bei der politische KandidatInnen in Reden und öffentlichen Dokumenten bestimmte Phrasen platzieren, die allein für weiße Evangelikale eine Bedeutung hätten und von anderen Wählergruppen ignoriert würden. Dafür müssen laut Calfano und Djupe zwei Voraussetzungen erfüllt sein: Erstens müssen die AdressatInnen die religiöse Sprache decodieren können, das heißt zum Beispiel Bibelzitate und -gleichnisse als solche identifizieren und ihre Bedeutung kennen. Zweitens muss den AdressatInnen die historische Unterstützung der Republikaner durch weiße Evangelikale bekannt sein. Das Experiment von Calfano und Djupe demonstriert, dass der Code durchaus seine Wirkung erfüllt. Weiße Evangelikale identifizierten einen fiktiven und einen entsprechenden Code verwendenden Kandidaten

4 Politische Heuristiken beinhalten auch weitere informationsreduzierende Elemente wie etwa Frames oder Primes. In welchem $\mathrm{Zu}-$ sammenhang etwa Frames zu ideologischen cues stehen, findet sich nicht nur in den englischsprachigen Arbeiten von Lau und Redlawsk (200I) sowie Jacoby (2000), sondern in der neueren deutschsprachigen Auseinandersetzung auch bei Schlipphak (20II). 
nicht nur durchschnittlich häufiger als Republikaner, sondern unterstützten ihn auch durchschnittlich stärker als Befragte anderer Konfessionen (Calfano/Djupe 2009, 329-331, 333-337).

Auf mögliche negative Effekte religiöser cues deuten demgegenüber die Erkenntnisse von McLaughlin und Wise (20I4) hin. Für ihren Versuch wurden Befragte mit den Botschaften fiktiver KandidatInnen für den USamerikanischen Kongress konfrontiert. Diese enthielten wahlweise keine oder eine von zwei möglichen Varianten eines religiösen cues. Sogenannte "overt cues" beinhalteten offene Bezüge auf Gott und das Christentum, während „subtle cues" lediglich eine spirituell aufgeladene Sprache nutzten, ohne sich konkret auf Gott oder das Christentum zu beziehen. Hierbei zeigte sich zum einen, dass unter allen Befragten die "overt cues“ im Durchschnitt negative Effekte auf die Beliebtheit und Wahlwahrscheinlichkeit der fiktiven KandidatInnen ausübten. Für beide Arten von religiösen cues wurde zum anderen getestet, ob sie mit der Religiosität der Befragten interagieren. Unter (sehr) religiösen Befragten resultierten sowohl subtile als auch offensichtliche cues in einer höheren Beliebtheit und Wahlwahrscheinlichkeit zugunsten der fiktiven KandidatInnen (McLaughlin/ Wise 20I4, 369-38I).

Diese Untersuchungen blieben aber bislang auf das US-amerikanische politische System beschränkt (McLaughlin/Wise 20I4, 368). Die Übertragbarkeit der Ergebnisse aus dieser Literatur auf den arabischen bzw. einen muslimischen Kontext ist daher nicht von vorneherein gegeben. In der Folge zeigen wir aber, wie sich die Erkenntnisse zur selektiven Wirkung religiöser cues mit den bisherigen Arbeiten zum politischen Vorteil islamistischer Parteien verbinden lassen. Dazu diskutieren wir zunächst das Argument des politischen Vorteils, bevor wir dieses Argument mit dem Modell der Nutzung von Heuristiken zusammenführen.

\subsection{Das Argument des politischen Vorteils islamisti-} scher Parteien und Hypothesenbildung

In ihrer Zusammenführung der bisherigen Literatur argumentieren Cammett und Luong (2014), dass sich der politische Vorteil islamistischer Parteien aus einer (wahrgenommenen) Trias aus Ideologie, organisatorischen Kapazitäten sowie karitativen Dienstleistungen ergibt. Erst das Zusammenwirken dieser Aspekte erzeuge die primäre Ursache für den politischen Vorteil, „a reputation for good governance“, das heißt „a reputation for being uniquely competent, trustworthy, and pure vis-à-vis the alternatives" (Cammett/Loung 20I4, I88f.).

Tatsächlich übt der Islamismus in arabisch-muslimischen Ländern seit Jahrzehnten eine Hegemonie gegenüber anderen Ideologien aus. Nicht zuletzt gilt das Scheitern alternativer, säkularer Ideologien als Entste- hungskontext des modernen Islamismus in den 1970er Jahren (Allani 2009, 259). Darüber hinaus bietet die Verankerung der Ideologie in religiösen Konzepten eine einzigartige Legitimationsgrundlage. Hierauf aufbauend sind IslamistInnen womöglich besonders effektiv im Erzeugen von Gruppenidentitäten und im Rahmen sozialer Probleme, das heißt der Einbettung dieser Probleme in ihren subjektiven Deutungsrahmen (Ayoob 2005, 952). Doch während die islamistische Ideologie das Potential bietet Massenzustimmung zu generieren, verweisen Cammett und Luong (2014, 198f.) darauf, dass Umfragen wiederholt gezeigt haben, dass nur eine Minderheit muslimischer AraberInnen die zentralen Ziele und Werte des Islamismus internalisiert hat.

Quantitativ kaum zu erfassen sei ferner das gängige Argument, dass IslamistInnen über das Erzeugen von Wohlfahrt Unterstützung generieren (so z.B. Woltering 2002, I034; Sadowski 2006, 226). Das liege vor allem daran, dass ForscherInnen häufig gar nicht ermitteln könnten, ob eine Einrichtung Verbindungen zu IslamistInnen unterhält. Entscheidend sei ohnehin nur, ob Teile der Bevölkerung glauben würden, dass IslamistInnen quantitativ und qualitativ mehr Wohlfahrt erzeugen als andere AkteurInnen. Eine Untersuchung von Tarek Masoud zeige, dass in Ägypten karitative Einrichtungen, die Namen tragen, die typischerweise mit IslamistInnen assoziiert werden, tatsächlich im Durchschnitt besser beurteilt werden. Das liege nicht zuletzt daran, dass IslamistInnen in glaubwürdiger Art und Weise auf die religiösen Konzepte "Zakat" und „Sadaqa“, das verpflichtende bzw. freiwillige Geben von Almosen, rekurrieren könnten (Cammett/Luong 20I4, I94f.).

Weitestgehend unumstritten ist laut Cammett und Luong die Überlegenheit der organisatorischen Kapazitäten von islamistischen Bewegungen. Das Alleinstellungsmerkmal bildet ihre Infrastruktur, die primär auf Moscheen, aber auch karitativen Einrichtungen und Koranschulen basiert. Mit diesen Einrichtungen ist zudem loyales Personal verbunden und die ProfiterInnen der angebotenen Leistungen stellen nicht selten lokale Unterstützernetzwerke her. Da die Führungsriegen islamistischer Organisationen sich häufig aus HochschulabsolventInnen zusammensetzen, verfügen sie zudem über technische Fähigkeiten und Management Know-how. Hinzu tritt die Finanzierung aus dem Ausland, allen voran aus den Golfstaaten. Doch eine Ideologie effektiv verbreiten zu können, sagt zunächst einmal nichts darüber aus, ob ihre zentralen Ideen, Werte und Ziele von den AdressatInnen auch internalisiert werden (Cammett/Luong 20I4, I94-197).

Der politische Vorteil islamistischer Parteien ergibt sich Cammett und Luong zufolge demnach aus der gleichzeitigen Wahrnehmung aller drei Aspekte, die mit dem islamistischen Parteilabel assoziiert würden: (I) eine konkurrenzlose Ideologie, die islamische Werte, 
wie Ehrlichkeit, Integrität und soziale Gerechtigkeit in den Mittelpunkt rückt; (2) organisatorische Kapazitäten, die eine effektive Verbreitung dieser Botschaften ermöglichen; sowie (3) karitative Einrichtungen, die in den sozioökonomisch schwachen Schichten der Bevölkerung den Botschaften von IslamistInnen Glaubwürdigkeit verleihen. Zusammen würden diese Aspekte für WählerInnen einen „informational shortcut about the type of moral character that candidates and parties are likely to embody if elected" (Cammett/Luong 20I4, 2OI) bereitstellen.

Mit dem islamistischen Parteilabel ist Cammett und Luong zufolge also eine Heuristik verbunden, über die WählerInnen die Erwartung ableiten, dass islamistische Parteien einen bestimmten, religiös begründeten und religiös aufgeladenen moralischen Gehalt haben, der auch die Umsetzung ihrer Politik bestimmt. Besondere Merkmale dieses moralischen Gehalts sind dabei - aus den drei Aspekten des politischen Vorteils abgeleitet - die (ideologisch bzw. religiöse begründete) Integrität und die (karitative und organisatorische) Kompetenz und Glaubwürdigkeit der Partei bzw. ihrer KandidatInnen. WählerInnen sollten also dann eine höhere Wahrscheinlichkeit haben, für islamistische Parteien zu stimmen, wenn ihnen die Integrität und die Glaubwürdigkeit des/der jeweiligen Kandidaten/-in wichtig sind.

H1: Je wichtiger die Integrität der politischen KandidatInnen für eine/n Befragte/n, desto höher die Wahrscheinlichkeit, dass der/die Befragte für die islamistische Partei stimmt.

H2: Je wichtiger die Glaubwürdigkeit der politischen KandidatInnen für eine/n Befragte/n, desto höher die Wahrscheinlichkeit, dass der/die Befragte für die islamistische Partei stimmt.

\section{Der Fall Tunesien - Fallauswahl und spezifische Fallerwartungen}

Um diese Hypothesen untersuchen zu können, muss eine zentrale Annahme erfüllt sein: Säkulare und islamistische Parteien müssen über formal gleiche Siegchancen verfügen. Das bedeutet, dass im untersuchten Land alle Parteien in fairen und freien Wahlen miteinander konkurrieren und nicht in der Äußerung von ideologischen Appellen eingeschränkt sein dürfen (Pepinsky et al. 20I2, 585). Unter den Ländern der „Arabellion“ erfüllt lediglich Tunesien für die vorliegenden Datensätze auf individueller Ebene diese Voraussetzung. Dass dort seit dem Regimesturz bereits mehrere Wahlen stattgefunden haben, begünstigt zudem die Diskussion der Untersuchungsergebnisse. Aus diesen Gründen beschränken wir unsere Untersuchung auf das tunesische Beispiel.
Analog zu den Erkenntnissen von McLaughlin und Wise (20I4) für die USA haben auch für Tunesien einige Autoren darauf hingewiesen, dass starke religiöse Bezüge Wähler eher abschrecken könnten. Dies liege darin begründet, dass das Ancien Régime seinen Medienapparat über Jahre hinweg darauf ausgerichtet habe, den Islamismus sowie eine besonders eifrige religiöse Betätigung im Allgemeinen mit Terrorismus gleichzusetzen (Boukhars 2015, II; Wolf 2013, 562). Tatsächlich hatten die Vorläuferorganisationen der islamistischen Ennahda über Jahre hinweg Schwierigkeiten, die breite Unterstützung der Bevölkerung zu gewinnen. Gleichzeitig gibt es jedoch auch Indizien, die dafür sprechen, dass politische Akteure in Tunesien um die Vorteile von subtilen religiösen cues wissen. Selbst die säkularen Diktatoren Habib Bourguiba und stärker noch Zine El Abidine Ben Ali griffen vereinzelt auf religiös-inspirierte Botschaften zurück und zwar immer dann, wenn es darum ging, ihre Herrschaft zu stabilisieren (Pargeter 2009, IO38; Wolf 2013, 562).

Ennahda selbst, so Cavatorta und Merone, konnte überhaupt erst an Popularität gewinnen, weil sie in den I990er und 200oer Jahren ihren ideologischen Kurs - und mit diesem das religiös-inspirierte Vokabular mäßigte. Ursächlich dafür waren die harte staatliche Repression einerseits und die mehrheitlich tolerantere Auslegung des Islams in Tunesien andererseits. In vier Bereichen werden die politisch-ideologischen Veränderungen besonders deutlich (Cavatorta/Merone 2013, 865-867).

Erstens akzeptierte die Partei aus taktischen Erwägungen bereits 1989 den Code du Statut Personell der zentrale Frauenrechte enthält. Zweitens gab sie das Ziel, einen islamischen Staat schaffen zu wollen, in dem die Scharia die alleingültige Rechtsgrundlage darstellt, vollends auf. In ihren offiziellen Stellungnahmen schreibt Ennahda der Religion nur noch eine identitätsstiftende Rolle zu und bekennt sich zur neuen demokratischen und pluralistischen Ordnung Tunesiens. Drittens verwarf Ennahda ihren scharfen Anti-Kapitalismus zugunsten einer wirtschaftsliberalen Politik. Auf diese Weise will Ennahda womöglich den Erfolg der türkischen AKP wiederholen, die den frommen Teil der Mittelschicht für sich gewinnen konnte. Viertens hat Ennahda ihren AntiAmerikanismus zugunsten einer stärker pragmatischen Außenpolitik zurückgefahren und rang sich Ende 2013 zu einer härteren Gangart gegenüber dschihadistischen SalafistInnen durch. (Cavatorta/Merone 2013, 860-862; Boukhars 2015, I3; Allani 2009, 264; Wolf 2013, 566; Guazzone 2013, 38f., 44f.; Roy 2012, 5If.).

Den Versuch, aus einer islamistischen Partei eine konservative Partei mit religiösen Bezügen zu machen, treibt vor allem Parteiführer Rachid Ghannouchi voran. Unter dessen Führung hat Ennahda ihre zentralen Versprechen eingelöst: Sie ging 20II eine Koalitionsre- 
gierung ein, akzeptierte, dass die neue tunesische Verfassung auf jeglichen Bezug zur Scharia verzichtet und stellte 2014 keine/n Präsidentschaftskandidaten/-in (Boukhars 2015, 14). Doch längst nicht alle EnnahdaMitglieder teilen Ghannouchis Kurs. Die Scharia-Debatte quittierten einige Mitglieder mit Parteiaustritten und zum Zeitpunkt der Datenerhebung (Februar 2013) gewann der dogmatische Parteiflügel stark an Zulauf (Wolf 2013, 566f.; Boukhars 2015, IO; Guazzone 2013, 45).

Die Positionierung der Ennahda hat damit zusammengefasst in den letzten Jahren an Komplexität gewonnen. Van Hamme et al. $(2014,754)$ zeigen jedoch, dass sich Ennahda nach wie vor entlang der modernistischkonservativen Konfliktlinie gegenüber den übrigen, säkularen Parteien deutlich abhebt. Auch heute noch geriert sich die Partei als "moral watchdog“ (Boukhars 2015, 6). Auch wenn beide Parteiflügel darüber streiten, wie weit die Islamisierung der Gesellschaft vorangetrieben werden sollte, stellt der Bezug auf religiös-konservative Werte weiterhin ihren gemeinsamen Nenner und Ennahdas Alleinstellungsmerkmal dar. Es ist daher am wahrscheinlichsten, dass die heutige Ideologie Ennahdas am ehesten die in islamischen Konzepten verankerten moralischen Qualitäten ihrer KandidatInnen signalisiert. Der jahrelange Kampf gegen das autokratische Ben-Ali-Regime verleiht dabei zusätzliche Glaubwürdigkeit.

\section{Daten und Operationalisierung}

\subsection{Arab Barometer Survey}

Um unsere Hypothesen zu überprüfen, verwenden wir in der Folge den Datensatz des Arab Barometer Survey. Dieser entstand im Jahr 2005 durch eine Kooperation von ForscherInnen der Universitäten Michigan und Princeton sowie Universitäten und Forschungszentren in der arabischen Welt. Durch diese Kooperation unterschiedlicher externer und regionaler SurveyspezialistInnen sowie durch die Abstimmung der Indikatoren mit den anderen Regionalbarometern unter dem Dach des Global Barometer Survey liefert das Arab Barometer als derzeit einziger Datensatz valide und reliable Indikatoren für unsere Argumentation. ${ }^{5}$ Die von uns genutzte dritte Welle des Arab Barometers ist mit Daten aus zwölf Ländern die bislang umfangreichste. In Tunesien fand die Datenerhebung im Februar 2013 - und damit vor den politischen Ereignissen im Land, die Ennahda unter innenpolitischen Druck setzten und eine Neuwahl erfor-

5 Weitere Surveys - wie etwa der PEW Gobal Attitudes Survey oder das European Neighborhood Barometer - haben ihre Schwächen darin, dass wichtige Indikatoren wie das Vertrauen in nationale AkteurInnen oder die Religiosität der Befragten nach 2007 nicht mehr oder grundsätzlich nicht abgefragt wurden. derlich machten (Guazzone 2013, 36-4I; Boukhars 2015, 3) - statt, wobei II99 Fälle mit einem Mindestalter von I8 Jahren erhoben wurden. ${ }^{6}$ Damit stellen alle zum Zeitpunkt der Erhebung in Tunesien lebende MuslimInnen ${ }^{7}$ mit einem Alter von mindestens I8 Jahren die Grundgesamtheit dar, auf die ausgehend von der Stichprobe rückgeschlossen werden soll.

\subsection{Operationalisierung des Messmodells}

Unsere abhängige Variable ist die Präferenz für eine islamistische beziehungsweise nicht-islamistische Partei. Im Arab Barometer wurden die Befragten um ihre Zustimmung zu den beiden folgenden Sätzen gebeten (Q605a im Fragebogen im Anhang):

„I prefer a religious political party over a non-religious political party."

„I prefer a non-religious political party over a religious political party."

Die Antwortoptionen sind dabei: starke Zustimmung zum ersten Satz, Zustimmung zum ersten Satz, Zustimmung zum zweiten Satz, starke Zustimmung zum zweiten Satz, keine Zustimmung zu einem der beiden Sätze und keine Ahnung / weiß nicht. Die Tatsache, dass in der Frage nach religiösen und nicht nach islamistischen Parteien gefragt wird, erscheint uns weitestgehend unproblematisch. ${ }^{8}$ Über eine Recodierung generierten

6 Dazu wurden standardisierte Fragebögen genutzt. Die Antworten der Befragten wurden von geschulten InterviewerInnen durch „face-to-face“ Gespräche - d.h., Gespräch von Angesicht zu Angesicht ohne Rückgriff auf Internet, Telefon etc. - auf tunesischem Arabisch ermittelt. Das komplexe Strichprobendesign baut auf einer mehrstufigen Zufallsstichprobe auf und verbindet sowohl Verfahren für geschichtete Stichproben als auch für Klumpenstichproben. Bei der Nutzung dieser Verfahren unterscheiden sich die Auswahlchancen der Fälle der Grundgesamtheit, in die Stichprobe aufgenommen zu werden. Deshalb wurden die Fälle entsprechend ihrer Auswahlchance gewichtet. Zudem wurde eine Nachgewichtung („post-stratification“) hinsichtlich Alter und Geschlecht vorgenommen, d.h., die Stichprobenverteilung wurde in diesen Punkten an die bekannte Verteilung der Grundgesamtheit angepasst (Arab Barometer 2014).

7 Obwohl in Tunesien auch AnhängerInnen religiöser Minderheiten leben, zeigt eine Ausgabe der Häufigkeiten für die Konfession der Befragten (QIOI2), dass sich alle Fälle der Stichprobe zum Islam bekennen.

8 Zwar wurden im Jahr 2012 vier weitere religiös-orientierte Parteien legalisiert, diese verfügen aber ebenfalls über eine islamistische Programmatik bzw. sind Neugründungen, die zum Zeitpunkt der Datenerhebung keine Möglichkeit hatten, sich (etwa durch Wahlen) der breiten Öffentlichkeit zu präsentieren. Die erste dieser Parteien, Tahrir, gilt gemeinhin als islamistisch, wenngleich sie sich durch ihre pan-islamistische Programmatik und ihre Zielsetzungen - Errichtung eines islamischen Staats und Verwirklichung der Scharia - von Ennahda unterscheidet. Die neugegründeten Parteien, Rahma, Asala und Jabhat Al Islah werden hingegen dem zeitgenössischen politischen Salafismus zugerechnet. Sie unterscheiden sich hinsichtlich ihrer ideologischen Wurzeln und inhaltlicher Schwerpunktsetzung. Ihnen ist allerdings gemein, dass sie die diversen islamischen Rechtschulen zu Gunsten einer textimmanenten Inter- 
wir eine Dummy-Variable, die jenen Befragten den Wert I zuweist, die dem ersten Satz zustimmten oder stark zustimmten, während Befragten, die der zweiten Formulierung zustimmten, der Wert o zugewiesen wird. Befragte, die keinem der beiden Sätze zustimmten oder keine Angabe machen wollten, wurden aus der Berechnung ausgeschlossen. Aufgrund der Operationalisierung der abhängigen Variablen als Dummy-Variable verwenden wir in der Folge ein logistisches Regressionsmodell.

Für die Operationalisierung der ersten Hypothese kann auf eine Variablenbatterie (Q520) zurückgegriffen werden. Hierbei wurden Befragte gebeten, sieben verschiedene Charakteristika von politischen KandidatInnen entsprechend ihrer Wichtigkeit zu sortieren. Dabei konnten die Befragten für jedes Charakteristikum einen Wert zwischen I (am wichtigsten) und 7 (am wenigsten wichtig) angeben. Unter den abgefragten Eigenschaften finden sich die beiden für die Hypothesen I und 2 relevanten Konzepte „Integrität“ und „vorherige politische Erfahrung". Für beide Konzepte wurden die Variablen so recodiert, dass nun höhere Werte eine höhere Zuschreibung an Relevanz der Konzepte durch die Befragten widerspiegeln. Während die Relevanzeinstufung von Integrität direkt mit der Erwartung in HI vereinbar ist, und wir daher eine positive Korrelation zwischen dieser unabhängigen und der abhängigen Variable erwarten, ist dies für das Konzept der politischen Vorerfahrung schwierig. Einerseits zielt das Konzept auf durch Erfahrung gewonnene Kompetenz ab. Andererseits, und das ist in Transformationsgesellschaften wie Tunesien besonders relevant, wird diese politische Vorerfahrung oftmals mit denjenigen Eliten zusammengebracht, die unter dem vorherigen autoritären Regime bereits Amtsträger waren. Da im Gegenzug die religiösen Parteien in dieser Zeit unterdrückt wurden, würde das in der $\mathrm{H}_{2}$ angelegte Glaubwürdigkeitsargument erwarten, dass WählerInnen, die unverbrauchte, glaubwürdige Akteure an der Macht sehen wollen, KandidatInnen ohne vorherige politische Erfahrung bevorzugen. Daher erwarten wir aus Sicht des Glaubwürdigkeitsarguments einen negativen Effekt der politischen Vorerfahrung auf die Wahl einer islamistischen Partei.

Die grundsätzliche Kontrollvariable für die Wahlwahrscheinlichkeit zugunsten religiöser Parteien sollte die Religiosität eines/r Befragten darstellen (Q609). Gerade in Zeiten der Unsicherheit über die tatsächlichen politischen Positionen von Parteien ist zu erwarten,

pretation des Koran ablehnen und die bestmögliche Nachahmung einer idealisierten Vergangenheit, angelehnt an die Zeit des Propheten und der ersten Folgegenerationen - den sogenannten „Altvorderen" (Al Salaf) -, anstreben. Beobachter beschreiben die drei Parteien zum Zeitpunkt der Datenerhebung als der tunesischen Bevölkerung weitestgehend unbekannt und auch in der Folgezeit blieben sie erfolglos. Weder Rahma, Asala und Jabhat Al Islah noch die Tahrir-Partei konnten bei den Parlamentswahlen 2014 einen Sitz gewinnen (ICG 2OI3, 2I-24; Marks 20I3, IO8f.). dass ein religiöses Parteilabel dazu führt, dass religiöse WählerInnen auch eher eine religiöse Partei wählen. Wir messen die Religiosität der SurveyteilnehmerInnen über deren Selbstauskunft. Gefragt nach der Ausprägung ihrer Religiosität konnten die Befragten unter den drei Antwortoptionen religiös, etwas religiös und nicht religiös wählen oder sich weigern zu antworten. Wir recodieren zunächst die Variable von $\mathrm{I}=$ nicht religiös bis 3 $=$ religiös und schließen alle Befragten, die eine Antwort verweigerten, aus der weiteren Analyse aus. Anschließend generieren wir zwei Dummy-Variablen, von denen die eine nicht religiöse Befragte $(=\mathrm{I})$ von allen anderen $(=0)$ trennt, während die andere alle religiösen Befragten $(=\mathrm{I})$ von allen weiteren $(=0)$ trennt. Als zusätzliche Kontrollvariablen ${ }^{9}$ beziehen wir zudem das Alter (QIooI) in Jahren, das Geschlecht (QIOO2) als Dummy (mit I= weiblich und $\mathrm{o}=$ männlich), einen Dummy für niedrige Bildung (mit $\mathrm{I}=$ alle Befragten, die keine oder nur eine Grundschule besucht haben, generiert aus QIoo3t), einen Dummy für höhere Bildung (mit I = alle Befragten, die einen tertiären Bildungsabschluss haben, generiert aus QIoo3t) sowie einen Dummy für den Wohnort des/ der Befragten (Q13), differenziert nach Stadt $(=0) /$ Land (= I), in das Modell mit ein. Dabei erwarten wir von der Stadt/Land-Variable sowie von den Bildungsvariablen einen substantiellen Effekt.

Die Annahme hinsichtlich der Stadt/Land-Variable beruht auf der historisch begründeten Unterstützung, die Ennahda in den ländlichen Gebieten im Süden und im Binnenland Tunesiens erfährt. Als ursächlich für dieses Phänomen gilt die Politik des Diktators Habib Bourguiba (Präsident von 1957-1987). Sein politisches Programm sollte Tunesien modernisieren und säkularisieren. Die Früchte dieser Politik ernteten aber größtenteils die mit seinem Regime verbundenen Eliten in den urbanen Küstenregionen des Nordostens. In der Folge konnte und wollte die Bevölkerung der politisch, sozial und ökonomisch vernachlässigten Regionen den Modernisierungstrends nicht folgen. Stattdessen wandten sich die BewohnerInnen dieser Landesteile verstärkt Denkrichtungen zu, die islamisch-konservative Werte hochhielten. Mit diesem Argument verknüpft ist schließlich auch die Erwartung, dass niedrige Bildung einen substantiellen Effekt zu Gunsten der Präferenz einer islamistischen Partei ausübt; erstens, weil in den

\footnotetext{
9 Wir verzichten an dieser Stelle auf den Einbezug weiterer Variablen, von denen gezeigt wurde, dass sie einen Einfluss auf das Wahlverhalten in Tunesien hatten, wie etwa die Nutzung sozialer Medien (vgl. etwa Ben M'Barek et al. 2015). Dies entspringt zwei theoretischen Überlegungen: Zum einen fokussieren wir uns nicht auf die vollständige Erklärung des Wahlverhaltens in Tunesien $(=\mathrm{Y})$, sondern sind am spezifischen Effekt bestimmter Heuristiken (=X) interessiert. Unser Ansatz ist daher $\mathrm{x}$ - und nicht y-zentriert. Zum anderen lassen sich im Hinblick auf die Wirkungskraft anderer Einstellungen immer auch Endogenitäts-Problematiken beobachten. So ist beispielsweise die Nutzung sozialer Medien nicht unabhängig von Alter und Bildung.
} 
vernachlässigten Regionen geringere Bildungschancen bestanden, was zweitens diejenigen, die eine bessere Bildung suchten, veranlasste, in die Städte abzuwandern (Pargeter 2009, I03I, I034-40).

Empirisch untermauert wird die Annahme einer sich geographisch manifestierenden modernistisch-konservativen Konfliktlinie dadurch, dass Ennahda in den Parlamentswahlen 2014 tendenziell mehr Stimmen erhielt, je südlicher das Wahlgebiet lag (Berman/Nugent 2015, 2). Aus diesen Gründen erwarten wir, dass ein ländlicher Wohnort auch einen signifikanten positiven Effekt auf den Wahlentscheid für eine islamistische Partei hat.

\section{Ergebnisse der empirischen Analyse}

\subsection{Deskriptive Statistiken}

Bei der Betrachtung der deskriptiven Statistiken zeigt sich für die abhängige Variable „Parteipräferenz", dass rund $60 \%$ der Befragten eine islamistische Partei gegenüber einer nicht-islamistischen Partei bevorzugen. Dies scheint angesichts der tatsächlichen Wahlergebnisse von Ennahda ein relativ hoher Anteil zu sein. Zum einen spiegeln aber unsere soziodemographischen Indikatoren eine der Gesamtbevölkerung sehr ähnliche Verteilung wider, sodass wir nicht von einem strukturellen Bias der Befragten ausgehen müssen. Zum anderen misst die abhängige Variable nicht direkt die (Nicht-) Stimmabgabe für Ennahda, sondern eine grundsätz- lichere Präferenz der Befragten. Hinsichtlich der unabhängigen Variablen aus HI und $\mathrm{H} 2$ zu den beiden Kandidateneigenschaften lässt sich aus der Tabelle ablesen, dass die beiden Charakteristika einerseits für die tunesischen WählerInnen im Durchschnitt relativ wichtig sind, es andererseits aber auch große Unterschiede zwischen den WählerInnen gibt.

In Bezug auf die Religiosität der untersuchten Fälle lassen die Häufigkeiten erkennen, dass nur $14 \%$ der Befragten sich selbst als nicht religiös eingestuft haben, während sich $36 \%$ als religiös einordnen. Während I6 \% der Befragten über eine tertiäre Bildung verfügen, haben fast die Hälfte der Befragten nur einen Grundschulabschluss oder gar keine formale Bildung vorzuweisen. Für die Stadt/Land- und Geschlechterverteilung spiegelt die Stichprobe annähernd die für die Grundgesamtheit bekannten Werte wider. Das Medianalter ist naturgemäß höher als in der Grundgesamtheit, da Unter-I8-Jährige von vornherein ausgeschlossen wurden (UN 20I4, 200; CIA 20I5).

\subsection{Erklärungsfaktoren für die Präferenz islamistischer Parteien}

In Tabelle 2 sind die Ergebnisse der logistischen Regression zur Erklärung der individuellen Präferenz zugunsten islamistischer Parteien in Tunesien abgetragen. Dabei haben wir für ordinal skalierte Variablen auf eine vereinfachte Berechnung und Darstellung zurückgegriffen, um einen besseren Überblick über die Effekte

Tabelle 1: Deskriptive Statistiken

\begin{tabular}{|c|c|c|c|c|c|}
\hline & $M W^{*}$ & Median & Min. & Max. & $\mathrm{StA}^{*}$ \\
\hline Präferenz islamistischer Parteien & 0.58 & 1 & 0 & 1 & 0.49 \\
\hline Vorherige politische Erfahrung & 5.04 & 5 & 1 & 7 & 1.77 \\
\hline Integrität & 4.60 & 5 & 1 & 7 & 1.56 \\
\hline Nicht religiös & 0.14 & 0 & 0 & 1 & 0.36 \\
\hline Religiös & 0.36 & 0 & 0 & 1 & 0.48 \\
\hline Alter & 43.6 & 42 & 18 & 87 & 15.83 \\
\hline Geschlecht & 0.50 & 1 & 0 & 1 & 0.50 \\
\hline Hohe Bildung & 0.16 & 0 & 0 & 1 & 0.37 \\
\hline Niedrige Bildung & 0.47 & 0 & 0 & 1 & 0.50 \\
\hline Wohnsitz auf dem Land & 0.35 & 0 & 0 & 1 & 0.48 \\
\hline
\end{tabular}

Quelle: Arab Barometer Wave 3. Eigene Berechnung.

MW = arithmetischer Mittelwert. StA = Standardabweichung. Min. und Max. = Beobachtete Minimal- und Maximalwerte.

* Für kategoriale Variablen dürfen Mittelwert und Standardabweichung strenggenommen nicht berechnet werden.

Sie geben aber dennoch - insbesondere im Fall der binären Variablen - einen grundlegenden Einblick in die Verteilung. 
Tabelle 2: Erklärungsfaktoren der Präferenz islamistischer Parteien

\begin{tabular}{lc}
\hline & Model 1 \\
\hline Konstante & $3.08(.46)^{\star * *}$ \\
\hline (H1) Integrität & $-.19(.05)^{\star * *}$ \\
(H2) Vorherige politische Erfahrung & $-.26(.05)^{\star * *}$ \\
\hline Religiös & $.41(.19)^{*}$ \\
Nicht religiös & $-1.00(.24)^{* * *}$ \\
Alter & $-.01(.01)$ \\
Geschlecht & $.14(.18)$ \\
Hohe Bildung & $-.11(.23)$ \\
Niedrige Bildung & $.44(.21)^{*}$ \\
Wohnsitz auf dem Land & $.46(.19)^{*}$
\end{tabular}

Anzahl Fälle / Pseudo R²

$951 / 10.0 \%$

Quelle: Arab Barometer Wave 3. Eigene Berechnung. ${ }^{* * *}=p<=.001,{ }^{* *}=p<=.01,{ }^{*}=p<=.05$.

Abbildung 1: Effektstärken von politischer Vorerfahrung und von Integrität

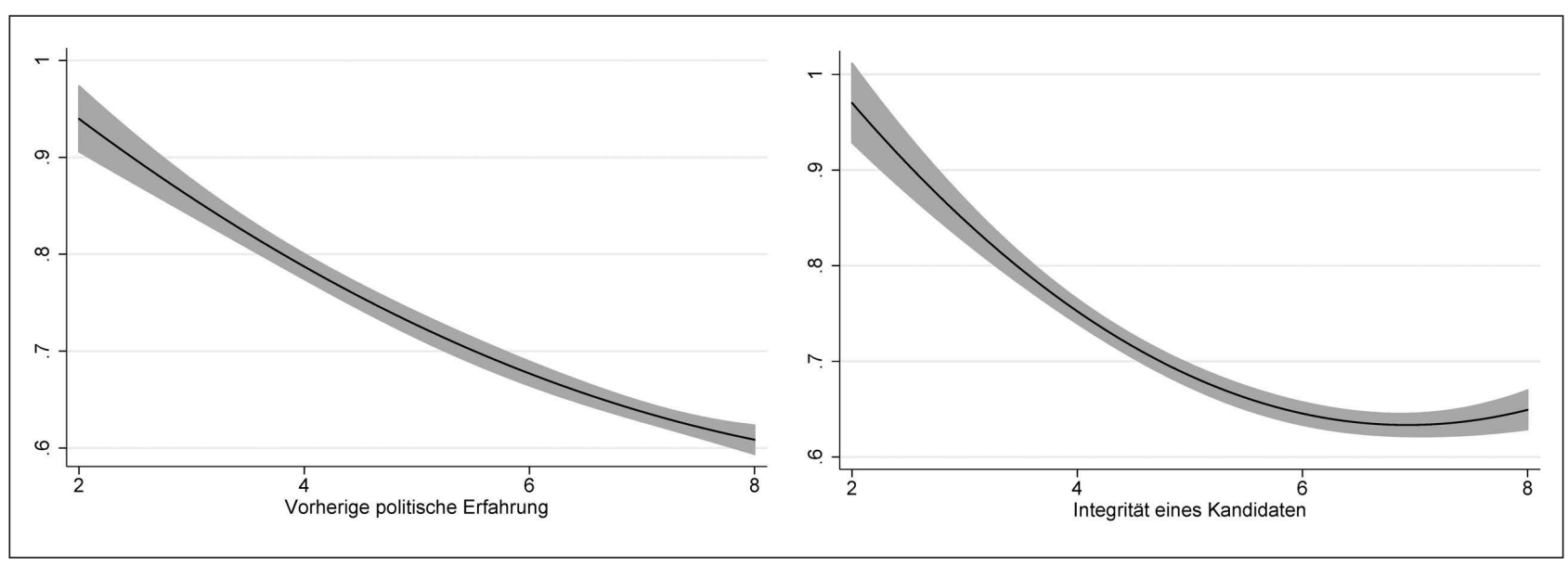

Quelle: Arab Barometer Wave 3. Eigene Darstellung.

der jeweiligen Variablen zu gewährleisten. Die korrekte Berechnung der Effekte der einzelnen Ausprägungsstufen für das Gesamtmodell findet sich in der Tabelle AI im Anhang und weicht nicht von den hier berichteten Haupteffekten ab.

Unsere Ergebnisse zeigen, dass alle in unseren Hypothesen enthaltenen Variablen einen signifikanten Einfluss auf die abhängige Variable aufweisen. Während wir für die vorherige politische Erfahrung auch das erwartete Vorzeichen beobachten können, weist Integrität im Gegensatz zu unserer Erwartung einen negativen
Effekt auf. Die Relevanz der Kandidatenintegrität führt tunesische WählerInnen also eher dazu, islamistische Parteien nicht zu wählen. Damit können wir an dieser Stelle $\mathrm{HI}$ als vorläufig widerlegt betrachten. Auch die Wichtigkeit der vorherigen politischen Erfahrung seitens des/der Kandidaten/-in hält die Befragten eher von der Wahl einer islamistischen Partei ab. Dies wurde von uns im Hinblick auf die speziellen Gegebenheiten in einem Transformationsstaat, in dem vorherige politische Erfahrungen meist auf eine systemunterstützende Funktion im alten (autoritären) Regime hinweisen, auch 
erwartet. Insgesamt lässt sich aufgrund dieser Ergebnisse die These vom politischen Vorteil islamistischer Parteien aufgrund ihrer ideologischen Integrität und sozialen Glaubwürdigkeit nur begrenzt bestätigen.

Wenig überraschend führt Religiosität dazu, dass Befragte eine wesentlich höhere Wahrscheinlichkeit aufweisen, islamistische Parteien zu wählen, als andere Befragte. Umgekehrt gilt dies in noch stärkerem Maße für nicht religiöse Befragte über alle Modelle hinweg.

Im Hinblick auf die Kontrollvariablen weisen nur zwei Variablen einen signifikanten Effekt auf: der Wohnort eines/r Befragten auf dem Land und die niedrige Bildung eines/r Befragten. Dieser Effekt kann als Bestätigung der oben diskutierten und auch in der Literatur nachgewiesenen Unterstützung der Ennahda durch große Teile der historisch marginalisierten, ländlichen Bevölkerung im Süden und im Binnenland Tunesiens einerseits und sozioökonomisch geringer ausgestatteten Teilen der Bevölkerung andererseits gelesen werden.

\subsection{Robustheit der Ergebnisse}

Um das Vertrauen in unsere Ergebnisse weiter zu erhöhen, führten wir einige Tests durch. Wir berechneten die Modelle nochmals mit robusten Standardfehlern. Dies führte zu keinen Veränderungen an den Ergebnissen. Weiter haben wir im Hinblick auf mögliche Multikollinearitätsprobleme eine Rangkorrelationsmatrix (Spearman) mit allen unabhängigen und Kontrollvariablen berechnet. Diese Berechnung erbrachte keinerlei auffällige oder problematische Befunde. Schließlich haben wir für die originale Skalierung der abhängigen Variablen in vier Ausprägungen ein „ordered logit“-Modell berechnet. Die Ergebnisse dieses Modells weisen darauf hin, dass der Einfluss der vorherigen politischen Erfahrung und der Nicht-Religiosität deutlich stärker ist als der Einfluss der Integritätsvariablen. Diese Ergebnisse wurden durch ein weitergehendes „ordered generalized linear model“ (oglm) (Williams 20IO) bestätigt, da im einfachen ordered logit-Modell die Annahme der parallelen Effekte verletzt wurde. Insgesamt zeigen alle Ergebnisse, dass der Wunsch nach der politischen Vorerfahrung eines/r Kandidaten/-in, die (Nicht-)Religiosität sowie der Wohnort eines/r Befragten einen verlässlichen und starken Einfluss auf die Präferenz einer islamistischen Partei aufweisen.

\section{Diskussion und Ausblick}

Existiert also - zurückkommend auf unsere Leitfrage ein politischer Vorteil islamistischer Parteien? Die Antwort ist aus unserer Sicht ein vorsichtiges, aber sicherlich weiter zu qualifizierendes Ja. Weder hat der Wunsch nach Integrität eines/r Kandidaten/-in einen über alle
Modelle hinweg signifikanten noch in der Richtung erwarteten Effekt auf die Präferenz einer islamistischen Partei. Zudem bleibt unsere Interpretation des Effektes, den der Wunsch nach der politischen Vorerfahrung eines/r Kandidaten/-in ausübt, nicht unumstritten. Weitere Forschung auf der Grundlage anderer Daten wird zeigen, ob sich die von Cammett und Luong theoretisch vermutete Kombination von ideologischer Integrität, überdurchschnittlicher Organisationskapazität und sozialer Glaubwürdigkeit tatsächlich in einem über Heuristiken vermittelten politischen Vorteil islamistischer Parteien auswirkt.

Weiter weisen die - von uns zum Teil ebenfalls aus der aktuellen Literaturdiskussion abgeleiteten - Kontrollvariablen (teilweise) einen signifikanten Einfluss auf die Wahrscheinlichkeit eines/r Befragten, eine islamistische Partei zu präferieren, auf. Angesichts des eindeutigen und einfach $\mathrm{zu}$ interpretierenden Effekts der Religiosität scheinen die Erkenntnisse von Pepinsky et al. (2012), wonach - zumindest im indonesischen Kontext - religiöse muslimische Wähler bei Unkenntnis über die konkreten politischen Positionen von Parteien tendenziell eher islamistische Parteien wählen, bestätigt zu werden. Noch stärker ist der Effekt der Nicht-Religiosität, der Befragte offenkundig dazu bringt, nichtreligiöse Parteien religiösen Parteien vorzuziehen und damit in die Richtung von McLaughlin und Wise (2OI4) deutet. Danach werden nicht-religiöse Wähler durch zu offenkundige religiöse Bezüge abgeschreckt.

Eindeutig zu interpretieren ist auch der signifikante positive Effekt eines ländlichen Wohnorts auf die Wahrscheinlichkeit, eine islamistische Partei zu präferieren. Der starke Einfluss des Stadt/Land-Prädiktors stützt die These von Pargeter (2009) sowie die Beobachtungen von Berman und Nugent (2015), dass tunesische IslamistInnen in den ländlichen Regionen des Südens und des Binnenlandes eine große Unterstützung generieren können. Dagegen variiert der Effekt der (niedrigen) Bildung von Befragten über die Modelle hinweg an Signifikanz, sodass auch an dieser Stelle zukünftige Forschung mit anderen Daten für eindeutigere Ergebnisse erforderlich erscheint.

Während wir mit diesen Erkenntnissen aus unserer Sicht einen wichtigen und ersten Schritt zur Überprüfung des politischen Vorteils islamistischer Parteien unternommen haben, müssen wir gleichzeitig angesichts der Komplexität der theoretisch hergeleiteten Konzepte, angesichts methodologischer Probleme und angesichts der Aktualität des Themas vorerst weitere wichtige Fragen unbeantwortet lassen. Erstens muss vorerst unklar bleiben, inwieweit die Relevanz der (Nicht-)Religiosität und moralischer Kandidatenspezifika angesichts der jeweiligen von den Parteien in den Vordergrund gerückten Themen im Wahlkampf variiert. Die Wahlergebnisse der Ennahda lassen zumindest vermuten, dass die Sali- 
enz von Themen einen Einfluss auf die Wirkung(skraft) der diskutierten cues haben. Darauf deuten die Ergebnisse der Meinungsumfragen von Berman und Nugent hin, die sie im Zuge der Legislativwahlen 201 I und 20I4 durchgeführt haben. Gegenüber 20II verloren die Themen Demokratisierung, Freiheitsrechte und "transitional justice" - also insgesamt der Bruch mit dem alten Regime - an Bedeutung. Wichtiger wurden den tunesischen Wahlberechtigen 2014 hingegen wirtschafts- und arbeitspolitische Themen (Berman/Nugent 2015, 7f.).

Zweitens bleibt offen, inwiefern die Ergebnisse auf andere nationale Kontexte und AkteurInnen übertragen werden können. Angesichts der Spezifika der Situation in Tunesien, die wir im Laufe des Artikels sowohl hinsichtlich der politischen Situation als auch der Entwicklung des tunesischen Islamismus mehrfach hervorgehoben haben, ist eine simple Verallgemeinerung unserer Ergebnisse über Tunesien hinaus natürlich unzulässig.

Drittens wird eine solche Verallgemeinerung dadurch eingeschränkt, dass die von uns verwendeten Indikatoren - aus unserer Sicht valide - „proxies“ für sehr komplexe theoretische Konzepte darstellen. Weitere Forschung in diesem Bereich, zu der wir auch in Zukunft beitragen wollen, steht daher vor der Herausforderung, Daten zu erheben und auszuwerten, die eine noch adäquatere Messung dieser Konzepte ermöglichen.

Sicher scheint hingegen, dass sowohl die Forschung als auch politische EntscheidungsträgerInnen von einer intensiveren Beschäftigung mit dem politischen Vorteil islamistischer Parteien profitieren könnten. Für die politische Verhaltensforschung bietet dieses Untersuchungsfeld die Möglichkeit zu testen, welchen Stellenwert politische Heuristiken bei der Formierung des Wahlentscheids in Ländern haben, die über keine demokratische Vorerfahrung verfügen. Vor allem aber stellt der politische Vorteil islamistischer Parteien ein neues Phänomen dar, das die Bedeutung der bisher im arabischen Kontext nur gering erforschten Heuristiken unterstreicht. In diesem Zusammenhang könnte auch der Untersuchung des (religiösen) Rahmens politischer Positionen und damit der Bedeutung von Forschung zu sozialen Bewegungen eine wesentlich größere Rolle zukommen (Snow et al. 1986). Diese Zusammenhänge weisen jedoch freilich über den Rahmen dieses Artikels hinaus. An unseren Erkenntnissen zeigt sich jedoch, dass eine Ausweitung des Analysefokus bisheriger Untersuchungen zu islamistischen Bewegungen und Parteien durchaus von Vorteil sein könnte. Anstatt allein die ideologische Entwicklung von Bewegungen und Parteien zu untersuchen, sollte auch die Wahrnehmung der Bevölkerung über diese ideologischen Positionen verstärkt in den Blick genommen werden.

Für politische EntscheidungsträgerInnen ergibt sich die Konsequenz, dass das Argument des politischen Vorteils keinen Grund für die Unterstützung säkularer au- tokratischer Regime darstellen sollte. Das Beispiel der tunesischen Ennahda zeigt deutlich, dass nur eine Mäßigung der ideologischen Positionen und das Verfolgen pragmatischer Wirtschafts-, Sicherheits- und Außenpolitiken eine Situation erzeugen kann, in der islamistische Parteien langfristig von ihrem politischen Vorteil profitieren können. Die Religion erfüllt dann auch weiterhin eine wichtige Funktion, dient aber stärker der Legitimation des politischen Programms und fungiert als identitätsstiftendes Element - ähnlich wie bei Europas Christdemokraten (Ozzano 20I3, 8II-8I4).

\section{Literatur}

Allani, Alaya (2009). The Islamists in Tunisia between Confrontation and Participation: 1980-2008, in: The Journal of North African Studies, Vol. I4(2), 257-272.

Arab Barometer (Hg.) (20I4). Codebook: Arab Barometer Wave III 20I2-20I4. Internet: http://www.arabbarometer.org/sites/default/files/code_book/Arab\%2O Barometer\%2oWave\%2oThree\%2oCodebook.pdf (Zugriff: 24.6.2016)

Ayoob, Mohammed (2004). Political Islam: Image and Reality, in: World Policy Journal, Vol. 2I(3), I-I4.

Ayoob, Mohammed (2005). The Future of Political Islam: the Importance of External Variables, in: International Affairs, Vol. 8I(5), 95I-96I.

Ben M'Barek, Melika/Nabil Jeddi/Mohamed Ali Achouri (2015). Impact of Social Media on the Behavior of Tunisian Voters: 2014 Elections, in: British Journal of Marketing Studies, Vol. 3(4), 32-44.

Berman, Chantal E./Elizabeth R. Nugent (2015). Defining Political Choices: Tunisia's Second Democratic Elections from the Ground Up, in: Brookings Center for Middle East Policy Analysis Paper, Vol. 38, I-29.

Boukhars, Anouar (2015). The Reckoning: Tunisia's Perilous Path to Democratic Stability, Washington, D.C.

Bustos, Rafael (2OII). Election Report: TUNISIA/Constituent Assembly Elections 23 October 20II. Internet: http:/opemam.org/sites/default/files/ER-Tunisia Constituent_assembly_20II.pdf (Zugriff: 24.6.2016)

Brown, Nathan J./Amr Hamzawy/Marina Ottaway (2006). Islamist Movements and the Democratic Process in the Arab World: Exploring the Gray Zones, in: Carnegie Papers, Vol. 67, I-I9.

Calfano, Brian R./Paul A. Djupe (2009). God Talk: Religious Cues and Electoral Support, in: Political Research Quarterly, Vol. 62(2), 329-339.

Cammett, Melani/Pauline J. Luong (20I4). Is There an Islamist Political Advantage?, in: Annual Review of Political Sciences, Vol. 17, 187-206.

Cavatorta, Francesco/Fabio Merone (2013). Moderation through Exclusion? The Journey of the Tunisian En- 
nahda from Fundamentalist to Conservative Party, in: Democratization, Vol. 2O(5), 857-875.

Central Intelligence Agency (Hg.) (2015). The World Factbook. Internet: https:/www.cia.gov/library/publications/the-world-factbook/geos/ts.html (Zugriff: 24.6.2016)

Denoeux, Guilain (2002). The Forgotten Swamp: Navigating Political Islam, in: Middle East Policy, Vol. 9(2), 56-8I.

The Economist (Hg.) (20II). Morocco's Election: Yet Another Islamist Victory. Internet: http://www.economist.com/node/2I54I058 (Zugriff: 24.6.2016)

Guazzone, Laura (2013). Ennahda Islamists and the Test of Government in Tunisia, in: The International Spectator: Italian Journal of International Affairs, Vol. 48(4), 30-50.

Hamid, Shadi (20II). The Rise of the Islamists: How Islamists Will Change Politics, and Vice Versa, in: Foreign Affairs, Vol. 90(3), 40-47.

International Crisis Group (Hg.) (2OI2). Lost in transition: The world according to Egypt's SCAF, in: Middle East Report, Vol. I2I, I-3I.

International Crisis Group (Hg.) (20I3). Tunisia: Violence and the Salafi Challenge, in: Middle East/North Africa Report, Vol. I37, I-53.

Jacoby, William G. (2000). Issue Framing and Public Opinion on Government Spending, in: American Journal of Political Science, Vol. 44(4), 750-767.

Lau, Richard R./David P. Redlawsk (200I). Advantages and Disadvantages of Cognitive Heuristics in Political Decision Making, in: American Journal of Political Science, Vol. 45(4), 95I-97I.

Lau, Richard R./David P. Redlawsk (2006). How Voters Decide: Information Processing during Election Campaigns, Cambridge.

Lau, Richard R./David O. Sears (1986). Social Cognition and Political Cognition: The Past, the Present, and the Future, in: dies. (Hg.): Political Cognition: The igth Annual Carnegie Symposium on Cognition, Hillsdale, NJ, 347-366.

Marks, Monica (20I3). Youth Politics and Tunisian Salafism: Understanding the Jihadi Current, in: Mediterranean Politics, Vol. I8(I), IO7-II4.

McLaughlin, Bryan/David Wise (20I4). Cueing God: Religious Cues and Voter Support, in: Politics and Religion, Vol. 7(2), 366-394.

Münch, Peter. 20I2. Bollwerk gegen den islamistischen Winter. Internet: http://www.sueddeutsche. de/politik/beziehungen-zwischen-aegypten-undisrael-bollwerk-gegen-den-islamistischen-winter-I.I393429 (Zugriff: 24.6.2016)

Ozzano, Luca (2013). The Many Faces of the Political God: a Typology of Religiously Oriented Parties, in: Democratization, Vol. 2O(5), 807-830.
Pargeter, Alison (2009). Localism and Radicalization in North Africa: Local Factors and the Development of Political Islam in Morocco, Tunisia and Libya, in: International Affairs, Vol. 85(5), IO3I-IO44.

Pepinsky, Thomas B./R. William Liddle/Saiful Mujani (2012). Testing Islam's Political Advantage: Evidence from Indonesia, in: American Journal of Political Science, Vol. 56(3), 584-600.

Phillips, Leigh (2011). Europe 'Should have Backed Democrats not Dictators,' Commissioner Says. Internet: http://euobserver.com/news/3I894 (Zugriff: 24.6.2016)

Popkin, Samuel L. (199I). The Reasoning Voter: Communication and Persuasion in Presidential Campaigns, Chicago, IL/London.

Rahn, Wendy M. (1993). The Role of Partisan Stereotypes in Information Processing about Political Candidates, in: American Journal of Political Science, Vol. $37(2), 472-496$.

Roy, Olivier (2012). Islamic Revival and Democracy: The case in Tunisia and Egypt, in: Olivier Roy/Cesare Merlini (Hg.): Arab Society in Revolt: The West's Mediterranean Challenge, Washington, D.C., 47-52.

Sadowski, Yahya (2006). Political Islam: Asking the Wrong Questions?, in: Annual Review of Political Science, Vol. 9, 2I5-240.

Schlipphak, Bernd (20II). Framing Ideology: Die Kommunikation ideologischer Positionierungen zwischen Parteien, Wählern und Medien, Baden-Baden.

Simon, Herbert A. (1957). Models of Man: Social and Rational, New York, NY.

Snow, David A./E. Burke Rochford/Steven K. Worden/ Robert D. Benford (1986). Frame Alignment Processes, Micromobilization, and Movement Participation, in: American Sociological Review, Vol. 5I(4), 464-48I.

Steinvorth, Daniel (2012). Islamists vs. Secularists: The Post-Revolution Struggle for the Arab Soul. Internet: http://www.spiegel.de/international/world/islamists-and-secular-society-battle-for-freedoms-after-arab-spring-a-870652.html (Zugriff: 24.6.20I6)

Süddeutsche Zeitung (Hg.) (20I4). Säkulare Partei gewinnt in Tunesien. Internet: http://www.sueddeutsche.de/ politik/amtliches-ergebnis-saekulare-partei-gewinnt-in-tunesien-I.2198057 (Zugriff: 24.6.2016)

United Nations (Hg.) (20I4). World Statistics Pocketbook 2014 edition, New York, NY.

Van Hamme, Gilles/Alia Gana/Maher Ben Rebbah (2014). Social and Socio-Territorial Electoral Base of Political Parties in Post-Revolutionary Tunisia, in: Journal of North African Studies, Vol. 19(5), 75I-769.

The Washington Times (Hg.) (2OII). From Arab Spring to Islamist Winter: Lack of U.S. Leadership Enables Radicalization in the Middle East. Internet: www. washingtontimes.com/news/20II/oct/25/from-arabspring-to-islamist-winter/ (Zugriff: 24.6.20I6) 
Williams, Richard (2010). Fitting Heterogeneous Choice Models with Oglm, in: The Stata Journal, Vol. Io(4), $540-567$.

Wolf, Anne (2013). An Islamist 'Renaissance'? Religion and Politics in Post-Revolutionary Tunisia in: Journal of North African Studies, Vol. I8(4), 560-573.

Woltering, Robbert A. F. L. (2002). The Roots of Islamist Popularity, in: Third World Quarterly, Vol. 23(6), II33-II43.

\section{Autoren}

Tim Lewis Poppenborg, geboren I99I, ist Absolvent der Universität Münster und studiert derzeit an der London School of Economics and Political Science (LSE). Zu seinen Interessen gehören Demokratisierungsprozesse sowie die Forschung zu politischen Bewegungen und bewaffneten Gruppen auf der Mikroebene.

Bernd Schlipphak, geboren 1979, ist Professor für empirische Methoden an der Universität Münster. Zu seinen Forschungsinteressen zählen politische Einstellungen in der arabischen Welt, die soziale Legitimität internationaler Organisationen sowie die vergleichende Regionalismusforschung. Neuere Publikationen zu diesen Themen erschienen in Review of International Organizations, European Union Politics und der Zeitschrift für Vergleichende Politikwissenschaft. 


\section{Anhang}

Tabelle A1: Formal korrekte Berechnung des Modells aus Tabelle 2

\begin{tabular}{|c|c|}
\hline & Coeff. (StE.) \\
\hline \multicolumn{2}{|l|}{ (H1) Integrität } \\
\hline 2 & $.52(.92)$ \\
\hline 3 & $-.39(.87)$ \\
\hline 4 & $-.23(.87)$ \\
\hline 5 & $-.88(.86)$ \\
\hline 6 & $-.57(.88)$ \\
\hline 7 (sehr wichtig) & $-.76(.87)$ \\
\hline \multicolumn{2}{|c|}{ (H2) Politische Vorerfahrung } \\
\hline 2 & $.80(.73)$ \\
\hline 3 & $-.89(.66)$ \\
\hline 4 & $-.65(.67)$ \\
\hline 5 & $-1.26(.64)^{*}$ \\
\hline 6 & $-1.22(.64)$ \\
\hline 7 (sehr wichtig) & $-1.35(.62)^{*}$ \\
\hline Religiös & $.44(.20)^{*}$ \\
\hline Nicht religiös & $-.96(.24)^{* \star *}$ \\
\hline Alter & $-.01(.01)$ \\
\hline Geschlecht & $.16(.18)$ \\
\hline Hohe Bildung & $-.09(.24)$ \\
\hline Niedrige Bildung & $.42(.22)^{*}$ \\
\hline Wohnsitz auf dem Land & $.49(.19)^{*}$ \\
\hline Konstante & $2.31(1.09)^{*}$ \\
\hline Anzahl Fälle / Pseudo $R^{2}$ & $951 / 11.8 \%$ \\
\hline
\end{tabular}

Quelle: Arab Barometer Wave 3. Eigene Berechnung. ${ }^{* * *}=p<=.001,{ }^{* *}=p<=.01,{ }^{*}=p<=.05$. 
Tabelle A2: Verwendete Variablen und ihre ursprüngliche Codierung

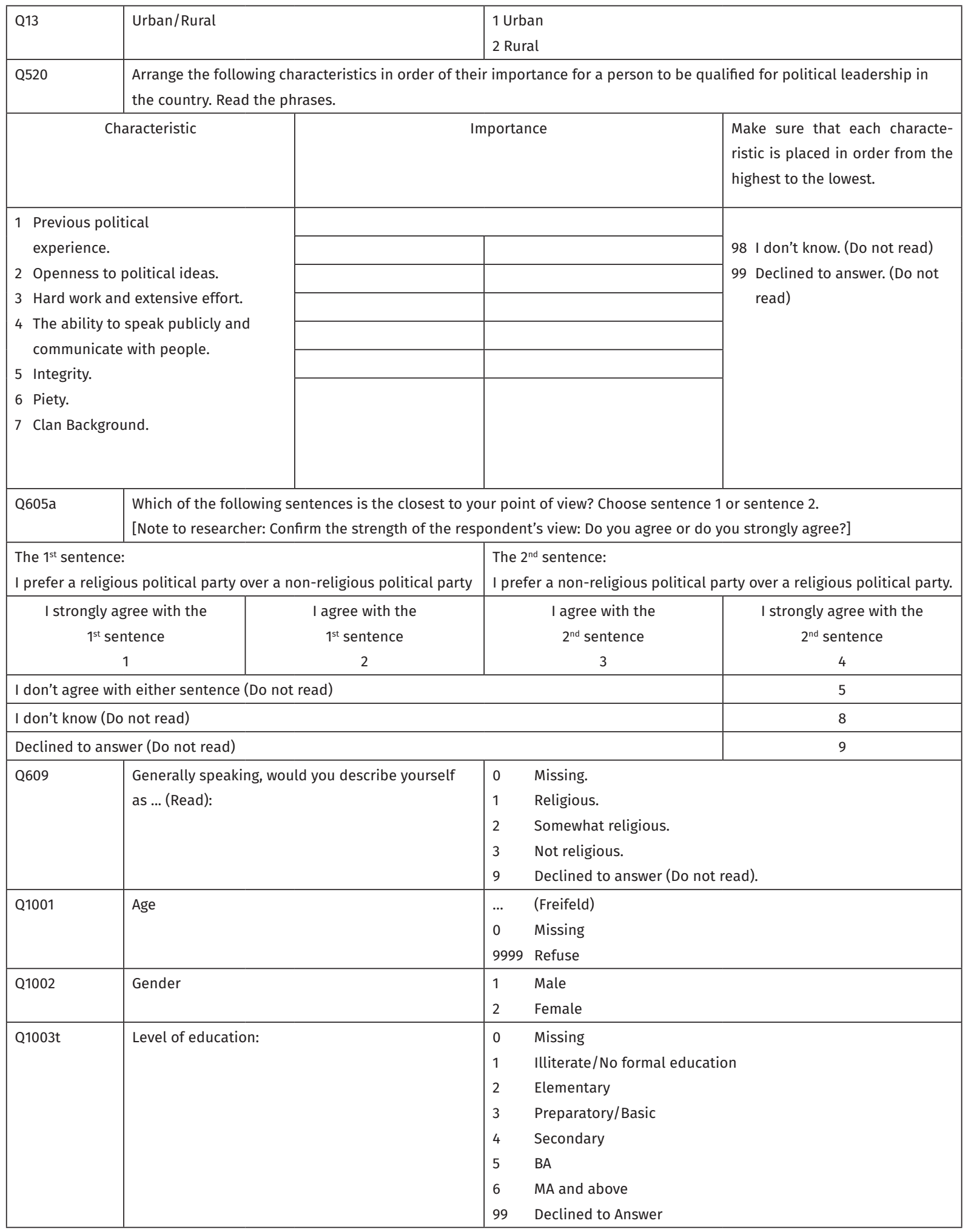


